\title{
Numerical Simulations for the Wake Prediction of a Marine Propeller in Straight Ahead Flow and Oblique Flow
}

\author{
E. Guilmineau*, G.B. Deng, A. Leroyer, P. Queutey, M. Visonneau, J. Wackers
}

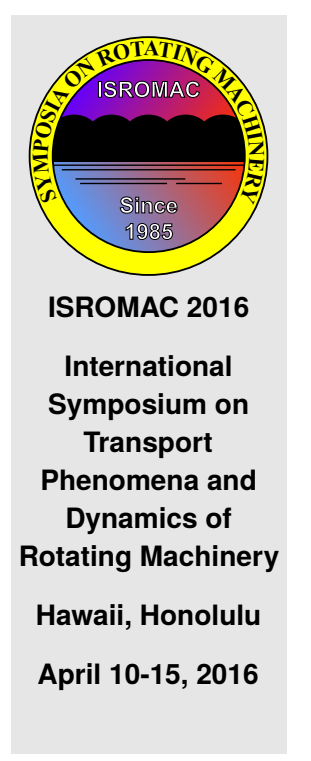

Abstract

This paper presents the capability of a numerical code, ISIS-CFD, based on the solution of the Reynolds averaged Navier-Stokes (RANS) equations, for the investigation on the hydrodynamics characteristics of a marine propeller in open water. Two propellers are investigated : the INSEAN E779A model in straight ahead flow and the PPTC model in oblique flow. The objectives of this study are to establish capabilities of various turbulent closures to predict the wake propeller, and to predict the instability processes in the wake if it exists.

Two RANS models are used: the k- $\omega$ SST of Menter and an anisotropic two-equation Explicit Algebraic Reynolds Stress Model (EARSM). A hybrid RANS-LES 5Large Eddy Simulation) model is also used. Comuptational results for global flow quantities are discussed and compared with experimental data. These quantities are in good agreement with the measured data.

The hydrid RANS-LES model allows to capture the evolution of the tip vortices. For the INSEAN E779A model, the instability of the wake is only predicted with a hybrid RANS-LES model and the position of these instabilities are in good agremment with the experimental visualizations.

Keywords

Marine propeller - Wake - Numerical simulation - ISIS-CFD — RANS — DES

LHEEA, Ecole Centrales de Nantes, Nantes, France

*Corresponding author: Emmanuel.Guilmineau@ec-nantes.fr

\section{INTRODUCTION}

The prediction of the fluid dynamics interaction between propellers and the hull is very important for the improvement of ship performance since the interaction is directly related to vibrations, noise and propulsion performances. In this context, the demand for the improvement of performance implies a rising interest in the development and application of detailed numerical tools.

The physical mechanisms that characterize the interaction between the propeller and the hull are very complex. However, even in the simpler case of an isolated propeller in a uniform flow, called open water conditions, we are confronted with several numerical and physical challenges. In the propeller field, a number of viscous phenomena can be identified including blade and hub boundary layers, flow separation on the blade, viscous-inviscid interaction, hub and tip vortices, viscous wake... Therefore, study of these flow characteristics is essential for accurate prediction of the propulsion performance. In this paper, we only focus on the open water conditions. With a pushing propeller in straight ahead flow, the flow is characterized by two vortex systems : one generated by the tip of the blade and the second emanated from the hub. A comprehensive description of the state of the art can be found in Felli et al. [1], who experimentally investigated the flow around a propeller in water tunnel. These authors studied the mechanisms that trigger the instability of wake and investigated the dependence of the vortex pairing and grouping on the mutual vortex distance.

Based on a numerical point of view, the reliability of the such numerical predictions can be questioned. It is difficult to control numerical diffusion when intense and localized three-dimensional structures are concerned. The flow in the core of the vortex is characterized by rotation, streamline curvature effects which are not adequately modeled by classical eddy-viscosity based turbulence models. Unsteady hybrid LES turbulence closures like DES appear attractive, see Muscari et al. [2].

Most studies only consider the case of a propeller in straight ahead flow. However, under real conditions, a working propeller operates behind a ship usually in a complex wake, so that the propeller shows quite different hydrodynamic performance. Moreover the consequence of the disturbance of the ship is that the angle of attack is different from blade to blade and the loads acting on the blades in axial direction is not symmetric.

Few authors have reported on hydrodynamic characteristics of a marine propeller in oblique flow. El Moctar and Bertram [3] used a Reynolds-averaged Navier-Stokes (RANS) solver to investigate the flow around a fourbladed modern propeller at oblique angles up to $12^{\circ}$. They found that the forces oscillate and the frequency of the oscillations increases with the angle of attack. Krasilnikov et al. [4] used an unsteady RANS method to investigate the blade forces acting on a podded propeller operating in oblique flow conditions. They found that blades of pulling propeller experience comparable ampli- 
tudes and load levels at positive and negative heading angles, being mainly affected by the cross-flow. The amplitudes and load levels on the blades of a pushing propeller are different at positive and negative headings due to the interaction of the propeller with the separated strut wake. Shamsi and Ghassemi [5] evaluated the performance of a podded propulsor in straight and azimuting condition by using a RANS approach with Moving Reference Frame. They found that the propeller thrust coefficient and the torque coefficient increase with increasing yaw angles. Their results also indicate that side force coefficients increase with increasing yaw angle and velocity advance ratio. Dubbioso et al. [6, 7] analyzed the performance of the INSEAN E779A propeller model in oblique flow by unsteady RANS and dynamically overlapping grid approach. Their main focus is on hydrodynamic loads that act on a single blade. They also discuss the flow features around the propeller. Unfortunately, due to the lack of experiments in oblique flow conditions, they did not perform a validation of the numerical computations. Yao [8] investigated the hydrodynamic performance of a 6 -bladed propeller in oblique flow. The hydrodynamic forces and moment showed a good agreement with experimental data under no cavitation condition or under weak cavitation condition.

The purpose of this paper is to conduct such a validation of the flow around an isolated propeller in straight ahead flow and oblique flow to compare statistical turbulence closures and an hybrid LES methodology to draw conclusions about the requirements in term of physics. The present paper is organized as follows. The test-cases are presented followed by the numerical method. The CFD results are presented for comparison and validation, as well as flow field analysis. Lastly, some concluding remarks are made.

\section{TEST-CASES}

In this paper, two propellers models are used: the INSEAN E779A model and the PPTC model.

\subsection{INSEAN E779A model}

The INSEAN E779A model is a four bladed, fixed-pitch, right-handed propeller characterized by a nominally constant pitch distribution and a very low skew angle. The diameter of the propeller is $\mathrm{D}=0.227 \mathrm{~m}$. The propeller is presented in Figure 1 and the main geometrical features are reported in Table 1.

In this paper, the rotational speed of the propeller is kept fixed to a value of $\mathrm{n}=25 \mathrm{rps}$ and the different advance coefficients $\mathrm{J}=\mathrm{U}_{\infty} /(\mathrm{nD})$ are obtained by changing the inflow velocity $\mathrm{U}_{\infty}$. The Reynolds number, Re $=1.78 \times 10^{6}$, is based on the radius of the propeller $(\mathrm{R}$ $=\mathrm{D} / 2=0.1135 \mathrm{~m})$ and the velocity of the tips of the blades $\left(\mathrm{U}_{\text {ref }}=\mathrm{n} \pi \mathrm{D} \approx 17.829 \mathrm{~m} / \mathrm{s}\right)$.

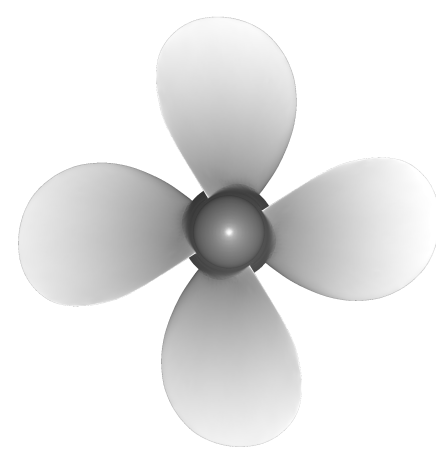

(a) Front view

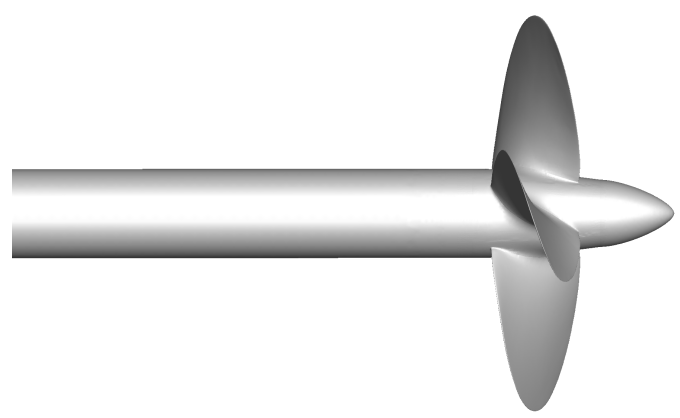

(b) Side view

Figure 1. INSEAN E779A model

Table 1. INSEAN E779A model: Geometric parameters

\begin{tabular}{ll}
\hline Pitch ratio & $\mathrm{P} / \mathrm{D}=1.10$ \\
Rake & $4^{\circ} 35^{\prime}$ \\
Expanded area ratio & 0.689 \\
Hub ratio & 0.200 \\
Skewed angle & $4.50^{\circ}$ \\
\hline
\end{tabular}

\subsection{PPTC model}

The Postdam Propeller Test Case (PPTC) model is a five bladed right-handed propeller which is a controllable pitch propeller designed to generate a stable tip vortex. The diameter of the propeller is $\mathrm{D}=0.250 \mathrm{~m}$. The propeller is presented in Figure 2 and the main geometrical features are reported in Table 2 . The blade 1 is the blade at 12 o'clock position in the initial configuration which corresponds to the angle $\theta=0$ degree.

In this study, the number of revolution per second is constant and is $\mathrm{n}=15 \mathrm{rps}$. The different advance coefficients, J, are obtained by changing the inflow ve-

Table 2. PPTC model: Geometric parameters

\begin{tabular}{ll}
\hline Pitch ratio & $\mathrm{P} / \mathrm{D}=1.635$ \\
Area ratio & 0.779 \\
Hub ratio & 0.150 \\
Skewed angle & $18.80^{\circ}$ \\
\hline
\end{tabular}




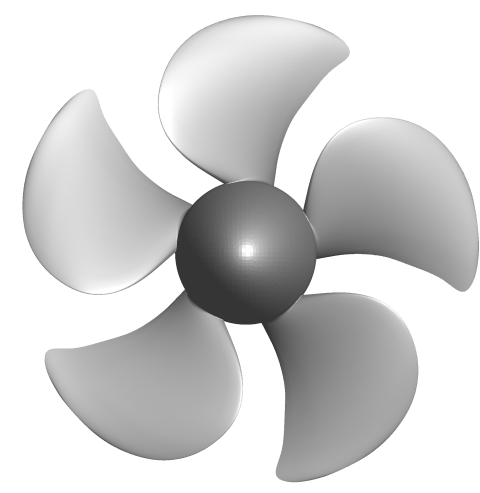

(a) Front view

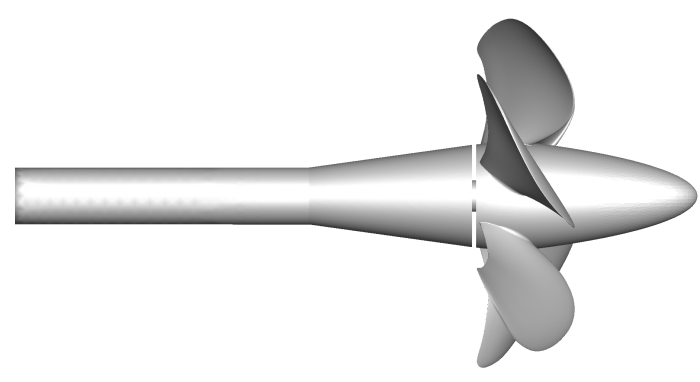

(b) Side view

Figure 2. PPTC model

locity. The Reynolds number, $\operatorname{Re}=1.39 \times 10^{6}$, is based on the radius of the propeller $(\mathrm{R}=0.125 \mathrm{~m})$ and the velocity of the tips of the blades $\left(\mathrm{U}_{\text {ref }}=\mathrm{n} \pi \mathrm{D} \approx 11.781\right.$ $\mathrm{m} / \mathrm{s})$. The propeller has an incidence inclination of 12 degrees towards the inflow direction.

\section{ISIS-CFD AT GLANCE}

The solver ISIS-CFD, available as a part of the FINE ${ }^{\mathrm{TM}}$ /Marine computing suite, is an incompressible unsteady Reynolds-averaged Navier-Stokes method mainly devoted to marine hydrodynamics. The method features several sophisticated turbulence models: apart from the classical two-equations $\mathrm{k}-\varepsilon$ and $\mathrm{k}-\omega$ models, the anisotropic two-equation Explicit Algebraic Reynolds Stress Model (EARSM), as well as Reynolds Stress Transport Models (RSTM), are available [9]. All models are available with wall-function or low-Reynolds near wall formulation. Hybrid LES turbulence models based on Detached Eddy Simulation (DES) are also implemented and have been validated on automotive flow characterized by large separation [10]. Additionally, several cavitation models are available in the solver.

The solver is based on finite volume method to build the spatial discretization of the transport equations. The unstructured discretization is face-based. While all unknown state variables are cell-centered, the system of equations used in the implicit time stepping procedure are constructed face by face and the contribution of each face is then added to the two cells next to the face. This technique poses no specific requirements on the topology of the cells. Therefore, the grids can be completely unstructured: cells with an arbitrary number of arbitrarily-shaped faces are accepted. Pressure-velocity coupling is enforced through a Rhie \& Chow SIMPLE type method: at each time step, the velocity updates come from the momentum equations and the pressure is given by the mass conservation law, transformed into a pressure equation. In the case of turbulent flows, transport equations for the variables in the turbulence model are added to the discretization.

Free-surface flow is simulated with a multi-phase flow approach: the water surface is captured with a conservation equation for the volume fraction of water, discretized with specific compressive discretization schemes [11]. The technique included for the 6 degrees of freedom simulation of ship motion is described by Leroyer \& Visonneau [12]. Time integration of Newton's law for the ship motion is combined with analytical weighted analogy grid deformation to adapt the fluid mesh to the moving ship. To enable relative motions of appendages, propellers or bodies without having recourse to overlapping grids, a sliding grid approach has been implemented. Propellers can be modeled by actuator disc theory, by coupling with boundary element codes (RANS-BEM coupling [13]) or with direct discretization through e.g. the rotating frame method or sliding interface approaches.

Finally, an anisotropic automatic grid refinement procedure has been developed which is controlled by various flow related criteria [14]. Parallelization is based on domain decomposition. The grid is divided into different partitions, which contain the cells. The interface faces on the boundaries between the partitions are shared between the partitions; information on these faces is exchanged with MPI (Message Passing Interface) protocol. The method works with the sliding grid approach and the different sub-domains can be distributed arbitrarily over the processors without any loss of generality. Moreover, the automatic grid refinement procedure is fully parallelized with a dynamic load balancing working transparently with or without sliding grids.

\section{NUMERICAL SIMULATION SET-UP}

For both cases, the computational mesh is created with Hexpress $^{\mathrm{TM}}$, an automatic unstructured mesh generator. This software generates meshes containing only hexaedrons.

\subsection{INSEAN E779A model}

For this model, a pusher configuration is investigated that means the hup cap is in the wake of the propeller. The computational domain consists of a cylindrical domain, whose the diameter is 3 times the propeller diameter, 


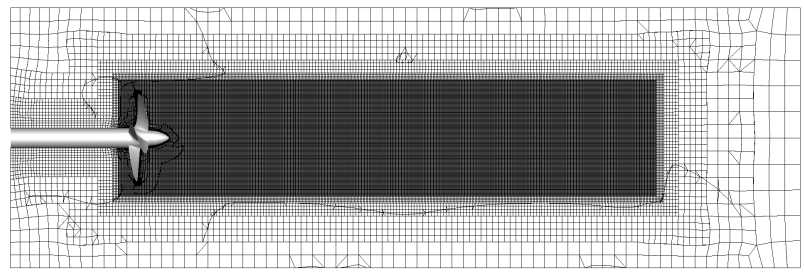

Figure 3. INSEAN model: View of the mesh in the plane $\mathrm{Y}=0$

and the length is 9.18 times the propeller diameter. It starts $3.96 \mathrm{R}$ before the propeller plane.

The mesh consists of $21.42 \times 10^{6}$ cells. The number of faces for each blade is approximately 38,100 . The average wall normal resolution on the blades is $\mathrm{y}^{+}=0.6$ with a maximum around the tip, in the order of 1.8. A box including the propeller and extending up to 6 diameters in the wake is added to capture the vortices. In this box, the cells are isotropic and the size is $0.0105 \mathrm{R}$, see Figure 3 .

For the RANS turbulence models (k- $\omega$ SST or EARSM), the solution is a steady solution while for DES computations, this approach is unsteady and the time step is $\Delta \mathrm{t}=$ $3 \times 10^{-5} \mathrm{~s}$ which corresponds to a rotation of 0.27 degrees. The time-averaged flow is obtained in approximately 9 rotations for DES computations.

\subsection{PPTC model}

For this model, the propeller is operating in a pull configuration with the hub cap pointing upstream. As the propeller is in incidence, in order to take into account the rotation of the propeller, the computational domain is decomposed in two regions: the rotating region close to the propeller and the stationary region. The total length of the computational domain is $15 \mathrm{D}$ with the inlet located 5D in front of the propeller rotation center. The computational domain has a width of $10 \mathrm{D}$ and a height of $6.5 \mathrm{D}$ with the top located $1.5 \mathrm{D}$ above the propeller rotation center.

The mesh consists of $28.74 \times 10^{6}$ cells with $28.25 \times 10^{6}$ cells in the rotating region. The number of faces for each blade is approximately 220,100 . The average wall normal resolution on the blades is $\mathrm{y}^{+}=0.3$ with a maximum around the tip, in the order of 2.0. In the rotating mesh, the cells are isotropic and the size is $0.025 \mathrm{R}$, see Figure 4. Figure 5 shows a partial view of the mesh in a plane $\mathrm{X}$ $=0$ which is the center of the propeller. The red circle in the figure represents sliding interfaces.

As the propeller is in incidence, a unsteady approach is used and the time step, for the RANS turbulence models, is $\Delta \mathrm{t}=1.889 \times 10^{-3} \mathrm{~s}$ which corresponds to a rotation of 1 degree while, for the hybrid RANS-LES, the time step is $\Delta \mathrm{t}=4.6296 \times 10^{-5} \mathrm{~s}$ which corresponds to a rotation of 0.25 degree.

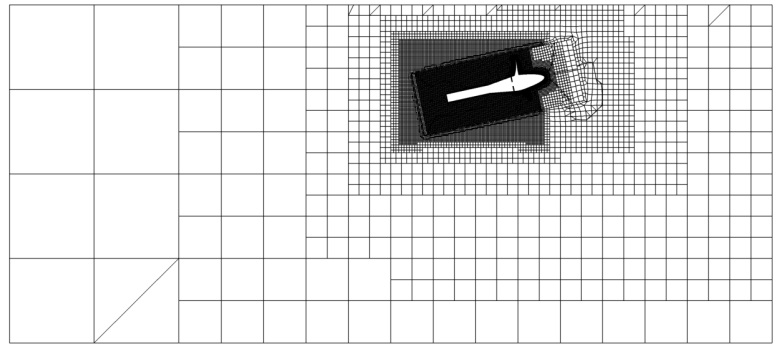

Figure 4. PPTC model: View of the mesh in the plane $\mathrm{Y}=0$

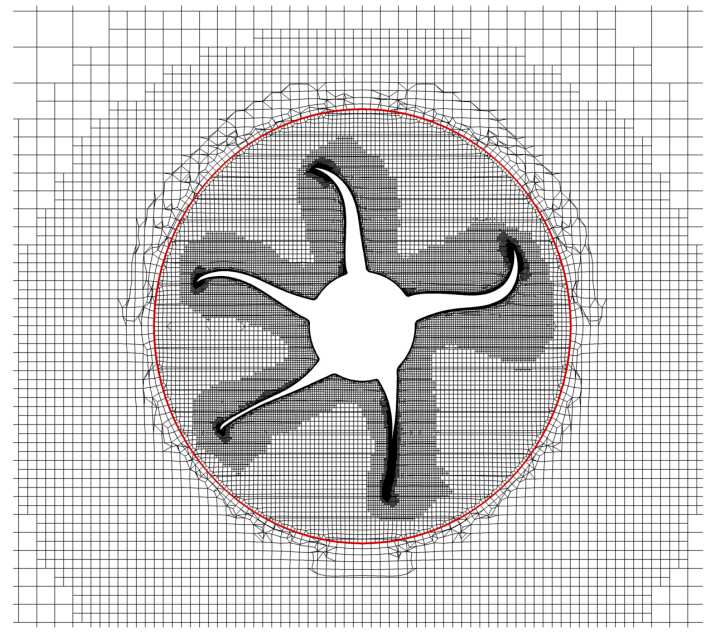

Figure 5. PPTC model: View of the mesh in the plane $\mathrm{X}=0$

\section{RESULTS AND DISCUSSION}

\subsection{INSEAN E779A model}

In order to compare different turbulence models for the prediction of the flow around the propeller, the open water characteristics of the model are investigated. Several numerical results for different values of the advance coefficient $J$ are compared with the experimental data [1], see Figure 6. Concerning the thrust coefficient $\mathrm{Kt}=$ $\mathrm{T} /\left(\rho \mathrm{n}^{2} \mathrm{D}^{4}\right)$, where $\mathrm{T}$ is the thrust of the propeller and $\rho$ the density of the water, the torque coefficient $\mathrm{Kq}=$ $\mathrm{Q} /\left(\rho \mathrm{n}^{2} \mathrm{D}^{5}\right)$, where $\mathrm{Q}$ is the torque, and the propeller open-water efficiency $\eta=\mathrm{Kt} / \mathrm{Kq} \times \mathrm{J} /(2 \pi)$, the predictions obtained with the different turbulence models are very similar and differ by less than $5 \%$ for the low values of the advance coefficient and by less than $3 \%$ for the high value of J. These numerical simulations are also in good agreement with the experimental data.

One of the objectives is to evaluate the ability of the turbulence model to reproduce some of the findings of Felli et al. [1]. The analysis of the flow field is carried out for two values of the advance coefficient, $\mathrm{J}=0.71$ and 0.45 .

A general view of the wake of the propeller, for the advance coefficient $\mathrm{J}=0.71$, is given in Figure 7 which 


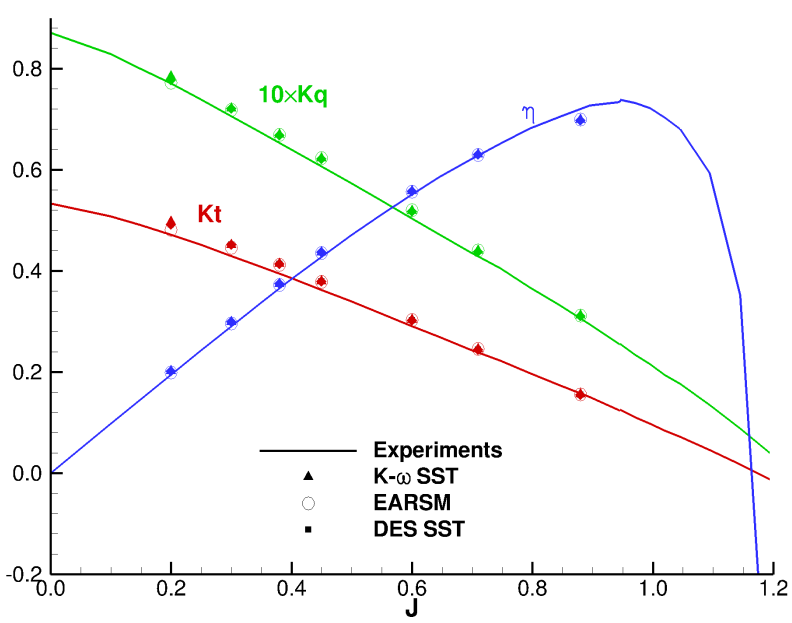

Figure 6. INSEAN model: Open-water characteristics

presents an isosurface of the dimensionless value $\lambda_{2}=$ -2 of the second largest invariant of $\mathbf{S}^{2}+\boldsymbol{\Omega}^{2}(\mathbf{S}$ and $\boldsymbol{\Omega}$ being the symmetric and antisymmetric component of $\nabla \mathbf{u})$ colored by the helicity. The acceleration of the flow behind the propeller causes a slight reduction of the radial position of the vortex cores. Then, the helices formed by the tip vortices remain located on a circular cylinder. RANS models yield tip vortices but they vanish more or less rapidly in the wake depending on the turbulence model used. With the DES approach, the tip vortices are maintained much further in the wake. These remarks are also observed by Muscari et al. [2]. Although no figure presents the turbulent kinetic energy (TKE), the RANS simulations predict a small value of TKE near the tip of the blade which grows rapidly in the vortex core. The values around the vortex core increase along the filament. On the contrary, the DES approach produces a high TKE near the tip but the level vanishes quickly in the wake and the level is lower than those observed with a RANS approach.

The detailed frequency analysis performed in the experimental work illustrates the process of energy transfer from the blade harmonic to the shaft at nearly at $\mathrm{x}=$ 7R [1], owing to vortex grouping. In the numerical simulation with the DES approach, the vortex grouping, marked by red ellipse, appears nearly $7.2 \mathrm{R}$, as shown Figure 8 . The instability of the propeller wake is characterized by a gradual deformation of the hub vortex and a sudden destabilization of the helical path of the tip vortices. The hub vortex describes a spiral geometry of progressively increased amplitude and the tip vortices tends to group where the hub vortex spiral is the local maximum.

Figure 9 illustrate a comparison of the velocity distribution in the wake of the propeller at $\mathrm{X}=110 \mathrm{~mm}$. The velocity is underestimated with the RANS simulations, see Figures 9(a) and 9(b) while the results obtained with the DES, see Figure 9(c), are in better agreement with

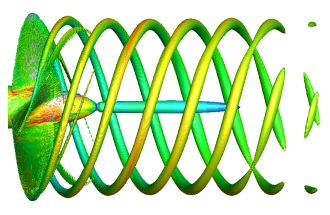

(a) k- $\omega$ SST

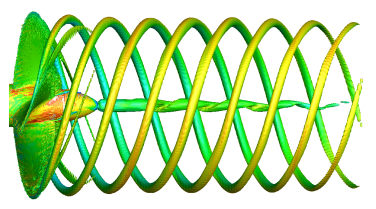

(b) EARSM

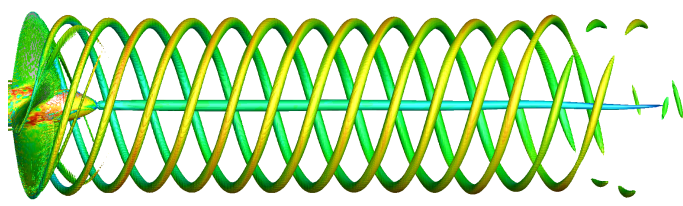

(c) DES

Figure 7. INSEAN model: $\mathrm{J}=0.71$ - Visualization of the vortical structures $\left(\lambda_{2}=-2\right)$

the experimental data, see Figure $9(\mathrm{~d})$. The maximum of velocity correspond to the position of the tip vortices. These areas are located at the same position for the numerical results while for the experimental data they are turned of few degrees.

These differences between the positions of the maximum of velocity are due to the spiral-to-spiral distance which is not similar, as in shown in Figure 10, which presents an instantaneous view of the vortical structure obtained with a DES approach and the visualization of Felli et al.(2011) [1] for another advance coefficient $\mathrm{J}=0.45$. These figures are very similar and present the pairing of the vortices. The difference between the numerical results and the experimental visualization is

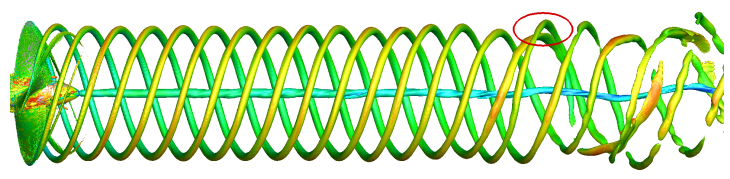

Figure 8. INSEAN model: $\mathrm{J}=0.71$ - Instantaneous visualization of the vortical structures with DES approach $\left(\lambda_{2}=-2\right)$ 


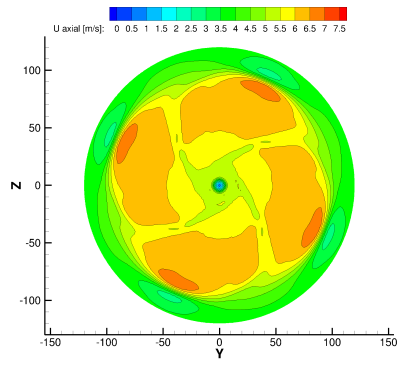

(a) k- $\omega$ SST

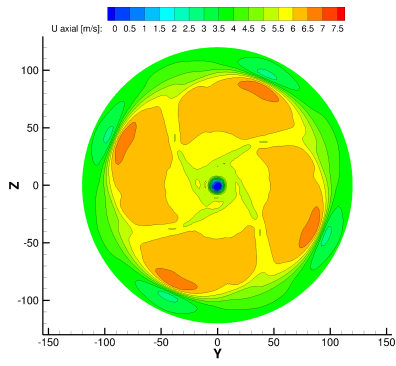

(b) EARSM

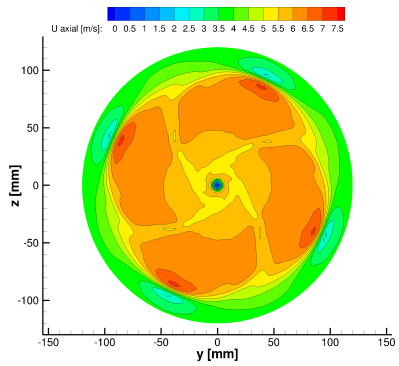

(c) DES

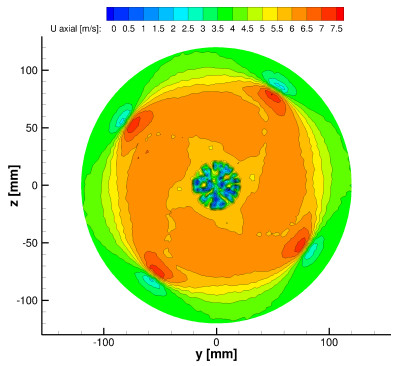

(d) Experiments [15]

Figure 9. INSEAN model: $\mathrm{J}=0.71$ - Axial velocity at $\mathrm{X}=110 \mathrm{~mm}$ in the wake of the propeller

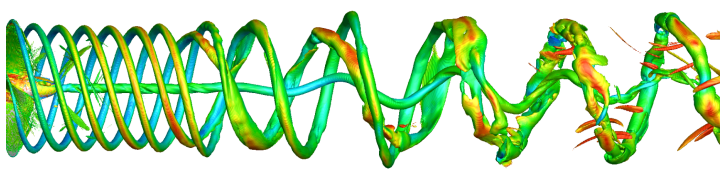

(a) DES (Instantaneous view)

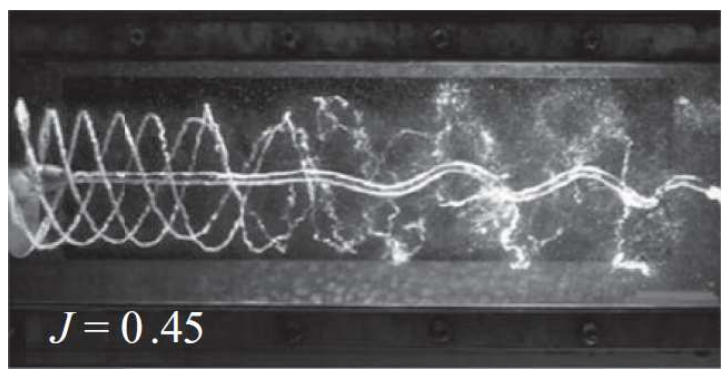

(b) Experimental view (Fig. 8 in Felli et al.(2011) [1]

Figure 10. INSEAN model: $\mathrm{J}=0.45$ - Visualization of the vortical structures $\left(\lambda_{2}=-2\right)$

the spiral-to-spiral distance. In the numerical simulation, this distance is shorter than that observed in experiments. However, the numerical spiral-to-spiral distance is in agreement with the numerical simulation of Muscari et al. [2] which used another flow solver with another mesh.

To compare all turbulence models for this advance coefficient, the averaged flow for the DES approach is presented with the RANS results in Figure 11. For the RANS results, the characteristics of the flow are the faster deformation of the wake and the stronger tip vortices. Even if the tip vortices are stronger than those predicted with $\mathrm{J}=0.71$, they are resolved over a short distance. This trend is also confirmed by another numerical results [2]. The DES method permits to predict more extended vortices and shows both the onset of the vortex instability and the start of the pairing process.

\subsection{PPTC model}

This propeller model has an inclination of 12 degrees with the upstream velocity. Thus the propeller performance

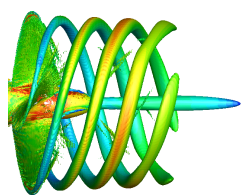

(a) $\mathrm{k}-\omega \mathrm{SST}$

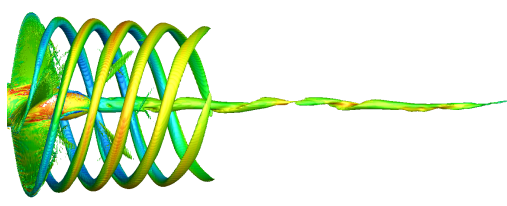

(b) EARSM

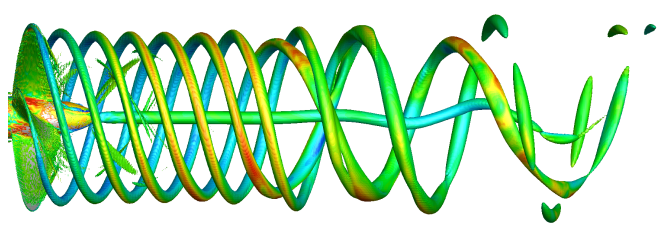

(c) DES

Figure 11. INSEAN model: $\mathrm{J}=0.45$ - Visualization of the vortical structures $\left(\lambda_{2}=-2\right)$

are evaluated in the Propeller Coordinate System (PCS), that is to say in the hub frame reference.

The open-water characteristics, obtained with the two RANS turbulence models and the hydrid RANS-LES model, are compared with the experimental data [16] in Figure 12. The agreement is the same order than the previous propeller model. The prediction of the thrust differ is overestimated by $3.2 \%$ for the lowest value of $\mathrm{J}$ and underestimated by $5.2 \%$ for the higher value of $\mathrm{J}$ while the torque coefficient is overestimated by $1.6 \%$ for the lowest value of $\mathrm{J}$ and underestimated by $6 \%$ for the highest value of $\mathrm{J}$.

As the propeller is in incidence, the loads generated 


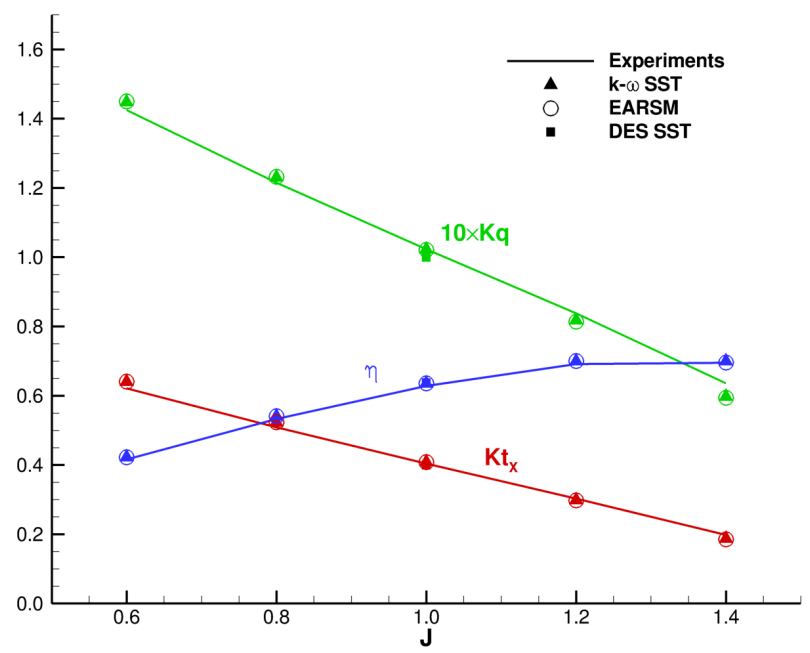

Figure 12. PPTC model: Open-water characteristics

by the blade are not constant during the revolution. This behavior can be evidenced in Figure 13, representing the forces and moments on the blade 1 in PCS. The mean value of these coefficients decreases as the coefficient $\mathrm{J}$ increases while the amplitude of these coefficients increases. It can be observed that the thrust, KTx, and the vertical force, $\mathrm{KTz}$, developed during the blade passage on the first half of the disk, $0^{\circ}<\theta<180^{\circ}$, are smaller, in absolute value, than those developed in the second half of the disk, $180^{\circ}<\theta<360^{\circ}$. The lateral force, $\mathrm{KTy}$, is phase-shifted with respect to the other ones by a quarter of cycle, i.e. $\pi / 2$. Similar comments hold for the moments.

A general view of the wake of the propeller, according to the advance coefficient $\mathrm{J}$, is given in Figure 14 which presents an isosurface of the dimensionless value $\lambda_{2}=$ -2 colored by the helicity obtained with the k- $\omega$ SST turbulence model. For all views, the blade 1 has the same position, the angle is 50.7 degrees but in this figure the blade 1 is represented at 12 o'clock. Some features of the tip vorticies are similar to the previous propeller model. As the advance coefficient increases, the size of the tip vortices are weaker and these vortices are more and more dissipated.

Figure 15 shows the influence of the turbulence modelization for the advance coefficient $\mathrm{J}=1.0$ at the angle $\theta=14.7$ degrees. In the figure, the blade 1 is located at 12 o'clock. As for the previous propeller, with the hybrid RANS-LES model, the tip vortices are maintained much further in the wake. For this numerical simulation, the tip vortices obtained with the DES is well predicted in the rotating region. then these vortices vanish because the mesh in the stationary region is not enough fine. The flow is very unsteady. When we compare the results for the same turbulence model but at two different instants, for example see Figure 15(a) and Figure 14(c), the wake of the propeller is very different. The separation, gen- erated at the foot of the blade, in the beginning of the motion disappears at the beginning of the second part of the motion.

The lateral vorticity, Y-component, for the advance coefficient $\mathrm{J}=1.0$, is presented, in the plane $\mathrm{Y}=0$, in Figure 16 according to the turbulence model used. The extreme values are more pronouced with the hydrid RANS-LES model than those obtained with the RANS model. Another difference is alos the wake of the shaft which interacts with the upper part of the tip vortices.

\section{CONCLUSIONS}

The capabilities of numerical simulations with various turbulence closures, RANS and DES, using ISIS-CFD flow solver, to predict the complex flow past an isolated propeller have been presented in this paper. Two propellers have been investigated: the INSEAN E779A model and the PPTC model. The first model is in straight ahead flow conditions while the second in oblique flow conditions.

For both propeller models, the numerical simulations, regardless of the turbulence model used, predict similar results for the global quantities, such as the thrust and the torque and they are in good agreement with the measured data. However, the prediction of the wake is not exactly the same, it depends on the turbulence model used. The hybrid RANS-LES approach allows to capture the evolution of the tip vortices if the mesh is fine enough. For the INSEAN E779A model, the DES model is able to predict the initial stages of the instability pattern with two consecutive filaments grouping their relative position, which agree reasonably well with the flow visualizations.

\section{ACKNOWLEDGMENTS}

This work was granted access to the HPC resources of CINES/IDRIS under the allocations 2014-2a0129 and 2015-2a0129 made by GENCI.

\section{REFERENCES}

[1] M. Felli, R. Camusi, and F. D. Felice. Mechanisms of evolution of the propeller wake in the transition and far fields. Journal of Fluid Mechanics, 682:5-53, 2011.

[2] R. Muscari, A. Di Mascio, and R. Verzicco. Modeling of vortex dynamics in the wake of a marine propeller. Computers \& Fluids, 73:63-79, 2013.

[3] O. El Moctar and V. Bertram. RANS simulation of propeller in oblique flow. In Proceedings of 3rd Numerical Towing Tank Symposium, pages 46-48, Tjärnö, Sweden, 2000.

[4] V. Krasilnikov, Z. Zhang, and F. Hong. Analysis of unsteady propeller blade forces by RANS. In 


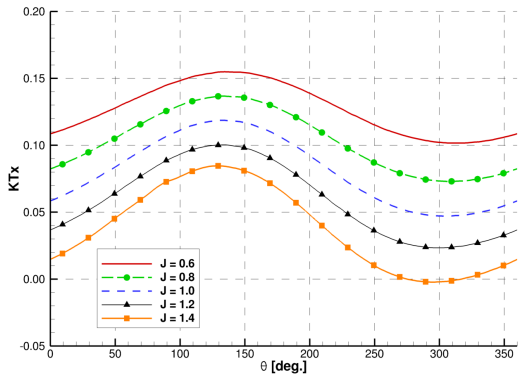

(a) KTx

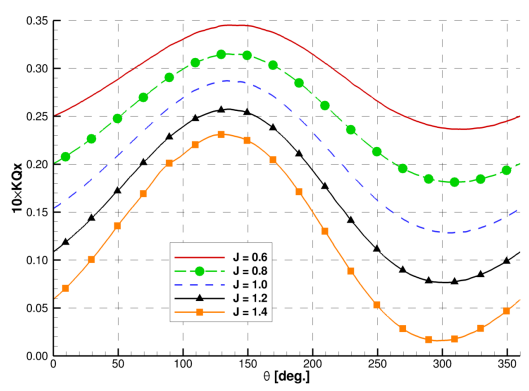

(d) KQx

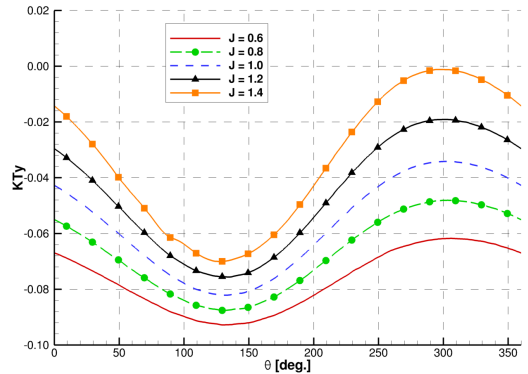

(b) KTy

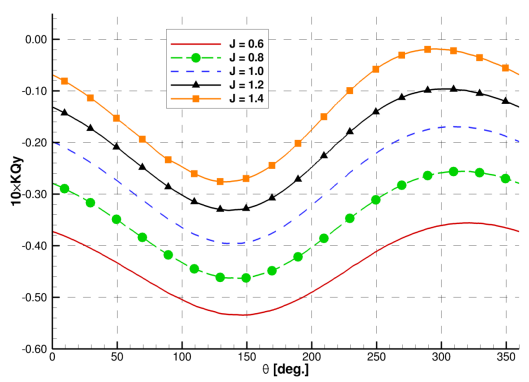

(e) KQy

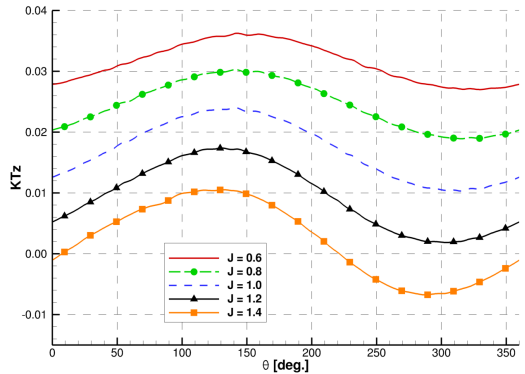

(c) $\mathrm{KTz}$

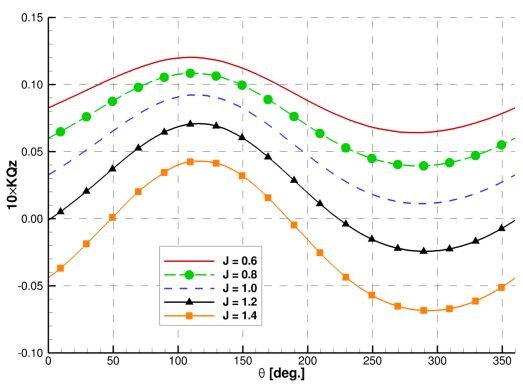

(f) $\mathrm{KQz}$

Figure 13. PPTC model: Force and moment coefficients on a single blade

First International Symposium on Marine Propulsors, SMP'09, pages 251-261, Trondheim, Norway, 2009.

${ }^{[5]}$ R. Shamsi and H. Ghassemi. Numerical investigation of yaw angle effects on propulsive characteristics of podded prouplsors. International Journal of Naval Archicteture and Ocean Engineering, 5:287301, 2013.

[6] G. Dubbioso, R. Muscari, and A. Di Mascio. Analysis of the performance of a marine propeller operating in oblique flow. Computers \& Fluids, 75:86-102, 2013.

[7] G. Dubbioso, R. Muscari, and A. Di Mascio. Analysis of the performance of a marine propeller operating in oblique flow. part 2: Very high incidence angles. Computers \& Fluids, 92:56-81, 2014.

[8] J. Yao. Investigation on hydrodynamic performance of a marine propeller in oblique flow by RANS computation. International Journal of Naval Archicteture and Ocean Engineering, 7:56-69, 2015.

[9] G. D. Deng and M. Visonneau. Comparison of explicit algebraic stress models and second-order turbulence closures for steady flow around ships. In 7th Symposium on Numerical Ship Hydrodynamics, pages 4.4-1-15, Nantes, France, July 1999.

[10] E. Guilmineau, G. B. Deng, and J. Wackers. Numerical simulation with a DES approach for automotive flows. Journal of Fluids and Structures, 27:807-816, 2011.
[11] P. Queutey and M. Visonneau. An interface capturing method for free-surface hydrodynamic flows. Computers and Fluids, 36:1481-1510, 2007.

[12] A. Leroyer and M. Visonneau. Numerical methods for RANSE simulations of a self-propelled fish-like body. Journal of Fluids and Structures, 20:975-991, 2005.

[13] G. B. Deng, P. Queutey, M. Visonneau, and F. Salvatore. Ship propulsion prediction with a coupled RANS-BEM approach. In B. Brinkmann and P. Wriggers, editors, MARINE 2013, International Conference on Computational Methods in Marine Engineering V, pages 541-551, 2013.

[14] J. Wackers, G. B. Deng, E. Guilmineau, A. Leroyer, P. Queutey, and M. Visonneau. Combined refinement criteria for anisotropic grid refinement in free-surface flow simulation. Computers \& Fluids, 92:209-222, 2014.

[15] M. Felli, G. Guj, and R. Camusi. Effect of the number of blades on propeller wake evolution. Experiments in Fluids, 44:409-418, 2008.

[16] S. Kinnas, M. Abdel-Maksoud, U. Barkmann, L. Lübke, and Y. Tian, editors. Proceedings of the Second Workshop on Cavitation and Propeller Performance - The Fourth International Symposium on Marine Propulsors, SMP'15, Austin, Texas, USA, 2015. 


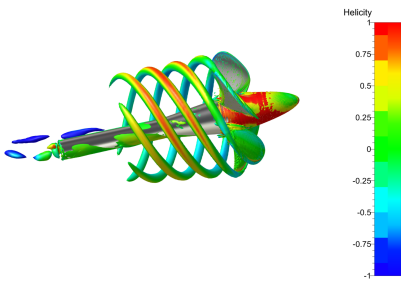

(a) $\mathrm{J}=0.60$

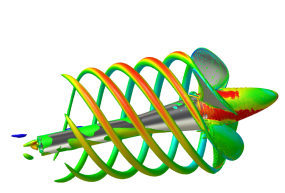

(b) $\mathrm{J}=0.80$

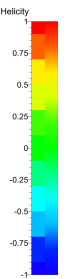

(d) $\mathrm{J}=1.20$
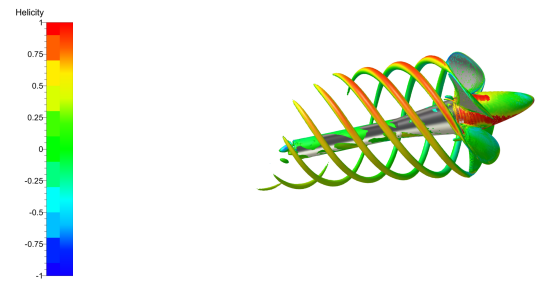

(c) $\mathrm{J}=1.00$

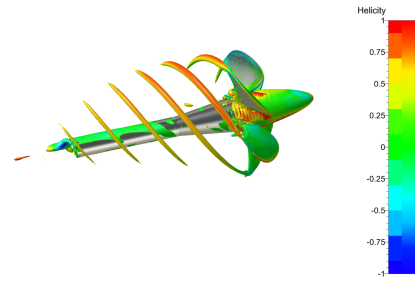

(e) $\mathrm{J}=1.40$

Figure 14. PPTC model: Visualization of the vortical structures $\left(\lambda_{2}=-2\right)$ at $\theta=50.7$ degrees with the k- $\omega$ SST turbulence model

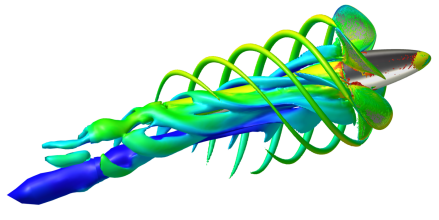

(a) $\mathrm{k}-\omega$ SST

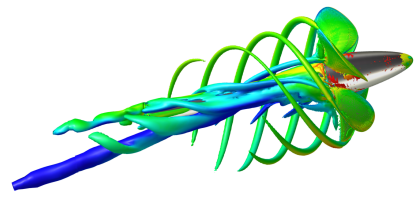

(b) EARSM

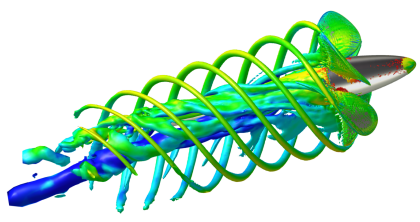

(c) DES
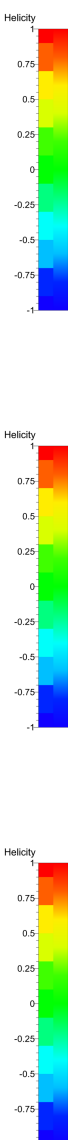

Figure 15. PPTC model: Visualization of the vortical structures $\left(\lambda_{2}=-2\right)$ for $\mathrm{J}=1.00$ at $\theta=14.7$ degrees

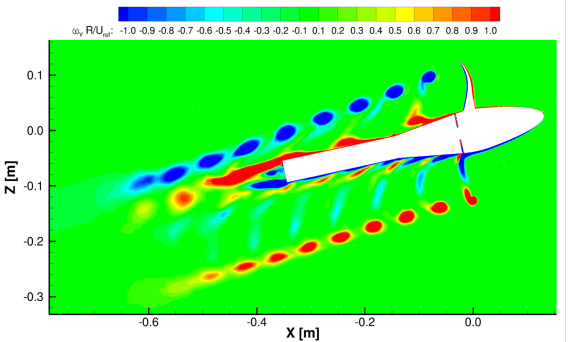

(b) EARSM

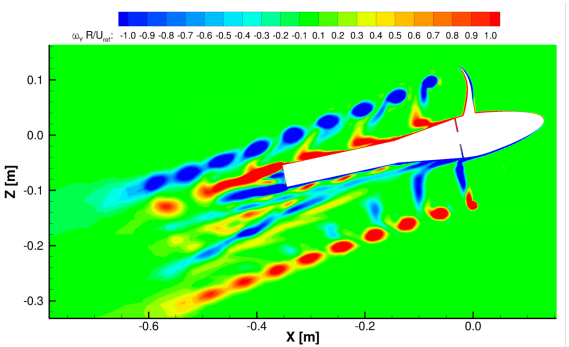

(a) k- $\omega$ SST

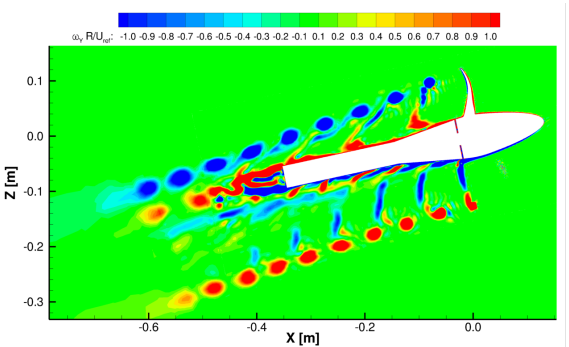

(c) DES
Figure 16. PPTC model: Vorticity in the plane $\mathrm{Y}=0$ for $\mathrm{J}=1.00$ at $\theta=14.7$ degrees 